\title{
Thiele, Alexander (Hrsg.) (2019): Legitimität in unsicheren Zeiten. Der demokratische Verfassungsstaat in der Krise?
}

\author{
Tübingen: Mohr Siebeck. 225 Seiten. 69,00€
}

\section{Peter Graf Kielmansegg}

Online publiziert: 23. April 2020

(C) Der/die Autor(en) 2020

Der demokratische Verfassungsstaat, die westliche Demokratie, wie man in Zeiten einer übersichtlicheren politischen Geografie sagte, ist zu einem Patienten geworden, dessen Zustand zur Besorgnis Anlass gibt - das ist inzwischen die herrschende Meinung, auch in der Politikwissenschaft. Dabei sind viele Diagnosen auf den Populismus als markantestes Krisenphänomen fixiert. Aber am Ende der Diagnose steht nicht selten die grundsätzliche Frage, ob die repräsentativ verfasste Demokratie überhaupt noch eine Zukunft habe. Auch der hier anzuzeigende Sammelband ist der Demokratiekrise auf der Spur. „Unsichere Zeiten“ - das meint Zeiten der Unsicherheit für die Demokratie. Seine Besonderheit liegt in der durch das Stichwort Legitimität vorgegebenen Perspektive.

Schon auf der ersten Seite präzisiert der Herausgeber Alexander Thiele die Fragen, um die es in seinem Band gehen soll: Was sind die Voraussetzungen, unter denen eine demokratische Ordnung als legitim angesehen werden kann? Woran lassen sich möglicherweise bestehende Legitimitätsdefizite festmachen? Wie lassen sich einmal erkannte Defizite wieder beseitigen?

Dieses Programm wird nach einer Einführung aus der Feder des Herausgebers, die dem Ganzen einen gedanklichen Rahmen gibt, in dreimal drei Beiträgen abgearbeitet, die sich den drei Ausgangsfragen zuordnen lassen. Im ersten Teil geht es um die theoretischen Grundlagen, das heißt im Kern um das Konzept der Legitimität, bezogen auf den demokratischen Verfassungsstaat; im zweiten um die empirische Frage nach den Legitimitätsdefiziten der Demokratie in ihrem gegenwärtigen Zustand; im dritten um die Möglichkeiten, diesen Defiziten abzuhelfen. In den zehn Beiträgen kommen unterschiedliche Fächer zu Wort, von der Rechtswissenschaft über die Politikwissenschaft und die Soziologie bis zur Wirtschaftswissenschaft. Das ist eine

P. Graf Kielmansegg $(\bowtie)$

Fakultät für Sozialwissenschaften, Universität Mannheim, Mannheim, Deutschland

E-Mail: petergrafkielmansegg@t-online.de 
Stärke des vorliegenden Bands. Es bedeutet aber auch, dass der Leser mit einer etwas verwirrenden Folge unterschiedlicher Perspektiven auf das gemeinsame Thema konfrontiert wird.

Der erste, theoretische Teil setzt mit einem ideengeschichtlichen Auftaktkapitel ein (Walter Reese-Schäfer), gibt dann der Rechtswissenschaft zur Explikation des juristischen Umgangs mit der Kategorie Legitimität das Wort (Friederike Wapler) und endet mit einem der wirtschaftswissenschaftlichen Debatte über Staatsversagen gewidmeten Beitrag (Achim Truger), der freilich nur einen sehr indirekten Bezug zum Thema Legitimität in seiner faktischen wie in seiner normativen Dimension hat.

Im zweiten, empirischen Teil erörtert Michael Kaeding, leider auf einer sehr schmalen Datenbasis, die Frage, was die deutliche soziale Asymmetrie in der Wahlbeteiligung für die Legitimität der Demokratie bedeutet; zeichnet Cornelia Koppetsch, wesentlich mit Befunden der Soziologie arbeitend, ein dramatisches Krisenpanorama, das die Demokratie im Zangengriff von Populismus und Plutokratie sieht; während Julian Krüper die Krise der Parteien diskutiert, die er zuerst als eine Krise der Maßstäbe, an denen Parteien gemessen werden, sodann als eine Krise des gesellschaftlichen Kontextes, in dem Parteien agieren, und erst an dritter Stelle als durch Leistungsschwäche der Parteien bedingt deutet.

Im dritten Teil schließlich, in dem es um Wege aus der Legitimationskrise geht, wird über die Neuordnung der sozialen Sicherungssysteme nachgedacht (Daniela Schweigler); über neue Formen dialogorientierter Bürgerbeteiligung, die deutlich von dem, was traditionell unter direkter Demokratie verstanden wird, unterschieden werden (Daniel Oppold und Patrizia Nanz); während ein letzter Beitrag unter der etwas kryptischen Überschrift „Legitimation durch Kopplung legitimatorischer Arenen“ an einem sehr spezifischen Beispiel, der Bedarfsplanung für die Stromnetze, untersucht, welche Legitimationsgewinne dadurch erreicht werden können, dass unterschiedliche Legitimationsprozesse in unterschiedlichen Politikarenen miteinander verknüpft werden (Simon Fink und Eva Ruffing).

Jeder wissenschaftliche Beitrag zur Legitimitätsthematik steht vor der schwierigen Aufgabe, einerseits kategorial zwischen der normativen Frage nach den guten Gründen für die Anerkennungswürdigkeit einer politischen Ordnung und der Faktenfrage nach der tatsächlichen Anerkennung einer politischen Ordnung als legitim unterscheiden, andererseits aber die normative und die empirische Dimension ständig zueinander in Beziehung setzen zu müssen. Was macht die Anerkennungswürdigkeit der Demokratie aus? Wie ist es um die tatsächliche Anerkennung einer bestimmten Demokratie als legitime Ordnung bestellt? Spielen die guten Gründe, auf denen die Anerkennungswürdigkeit beruht, für die tatsächliche Anerkennung, die ja auch ganz andere Wurzeln haben kann, eine Rolle? Diese drei Fragen müssen sorgfältig auseinandergehalten und doch auch wieder ständig aufeinander bezogen werden. Es ist unvermeidlich, wenn auch nicht wirklich befriedigend, dass jeder Beitrag sein eigenes Verständnis von dieser Aufgabe hat. Der Herausgeber gibt zwar das empirische Verständnis von Legitimität - soziale Anerkennung einer bestehenden Herrschaftsordnung als grundsätzlich gerecht durch die Herrschaftsunterworfenen als das für den Band maßgebliche vor. Und diese Vorgabe wirkt auch in den Band hinein, aber von Beitrag zu Beitrag doch in sehr unterschiedlichem Maße. 
Sieht man die Fragen, ob die Anerkennung des demokratischen Verfassungsstaats als legitim durch seine Bürger tatsächlich erschüttert sei und wie, wenn ja, dem entgegengewirkt werden könne, als die Kernfragen von Thieles Band an, kommt den Beiträgen von Koppetsch und Schweigler eine Art von Schlüsselbedeutung zu. Der eine verficht am entschiedensten die These, dass wir es mit einer Legitimitätskrise der Demokratie zu tun haben, einer Krise, die letztlich in wachsender sozialer Ungleichheit ihre Wurzeln hat. Der andere entwirft ein Programm umfassender Reformen der sozialen Sicherungssysteme, um dieser Krise zu begegnen. Der These, dass wachsende Ungleichheit (oder auch wachsende Sensibilität für Ungleichheit) Demokratien in eine Legitimitätskrise gleiten lassen kann, wird kaum jemand widersprechen. Auch die Vermutung, dass Demokratien, was immer sie sonst für Vorzüge haben mögen, von ihren Bürgern vor allem daran gemessen werden, ob sie Status- und Einkommenssicherheit gewährleisten können, ist plausibel. Allerdings hätte man sich gewünscht, dass die Frage, ob demokratiespezifische Legitimitätsquellen zumindest eine sekundäre Rolle für die faktische Anerkennung von Demokratien als legitim spielen, mehr Aufmerksamkeit gefunden hätte.

Viel weniger einsichtig ist dann aber die dramatische Diagnose, dass die bundesrepublikanische Demokratie - beide Texte haben nicht ausschließlich aber ganz überwiegend sie im Visier - in eine Legitimitätskrise geraten sei, weil sie soziale Sicherheit nicht mehr garantiere, sondern Marktradikalismen freien Raum gebe, die zu unerträglichen Ungleichheiten führten. Im Zentrum von Koppetschs Analyse steht die These, dass der Mittelstand, den sie als eigentlichen Träger der Demokratie ansieht, schrumpfe. In einem unhistorisch scharfen Kontrast stellt sie die alte Bundesrepublik, die eine harmonische Mittelstandsdemokratie gewesen sei, der neuen Bundesrepublik, in der die entfesselten Marktkräfte den sozialen Zusammenhalt zerstörten, gegenüber. Die Folgen dieser Entwicklung werden in drastischen Pauschalurteilen beschrieben: Die Mittelschicht besitze heute keinen nennenswerten Einfluss mehr auf die gesellschaftliche und wirtschaftliche Entwicklung des Landes. Der Wohlstand sinke für die Mehrzahl der Arbeitnehmergruppen. Der Sozialstaat werde abgebaut. Geburt und Herkunft entschieden stärker denn je - man höre und staune: stärker denn je! - über Lebenschancen.

Der Leser tut sich mit diesen Urteilen schon deshalb schwer, weil Koppetsch ihm nie verrät, was genau sie unter Mittelschicht versteht. Vor allem aber hätte er sich entschieden mehr über bloße Krisenessayistik hinausführende, datengestützte sozialwissenschaftliche Genauigkeit gewünscht.

Schweigler überführt diese Krisendiagnostik in eine umfassende Wunschliste sozialpolitischer Reformen. Ein solches Wunschprogramm entwirft sich leicht, wenn die Frage gar nicht ins Blickfeld gerät, unter welchen Bedingungen eine im globalen Wettbewerb stehende Wirtschaft ein Sozialprodukt erwirtschaften kann, das dieses Programm zu finanzieren vermag. Dass die Bürgerversicherung und ein allgemeines Grundeinkommen dazu gehören, überrascht nicht. Darüber kann man diskutieren. Aber auch hier wünscht man sich mehr sozialwissenschaftliche Disziplin des Argumentierens, eine Disziplin, die auch dann noch durchgehalten wird, wenn sie den eigenen politischen Präferenzen in die Quere kommt. Zur Aussage, dass die Armutsquote kontinuierlich steige, gehört doch wohl ein Hinweis darauf, dass hier von relativer Armut die Rede ist - und selbst für relative Armut ist die Aussage 
nicht korrekt. Wer die Rentenprobleme thematisiert, sollte nicht verschweigen, dass sie ihre Wurzeln vor allem in der demografischen Entwicklung und nicht im bösen Willen von Politikern haben. Aber wer schon die Aufforderung, auch privat für das Alter vorzusorgen, für repressive Sozialpädagogik hält, sieht das offenbar anders.

Sein Band, so formuliert Thiele es im Vorwort, solle einen Beitrag zu einer ,stetigen Debatte“ (S. vii) leisten, die nicht abschließbar sei. Dass wir derzeit besonderen Anlass haben, darüber nachzudenken, wie es um die Legitimität des demokratischen Verfassungsstaats bestellt sei, steht außer Frage. Man muss nicht jedes Kapitel für gelungen halten, um den Band als einen solchen Beitrag willkommen zu heißen.

Funding Open Access funding provided by Projekt DEAL.

Open Access Dieser Artikel wird unter der Creative Commons Namensnennung 4.0 International Lizenz veröffentlicht, welche die Nutzung, Vervielfältigung, Bearbeitung, Verbreitung und Wiedergabe in jeglichem Medium und Format erlaubt, sofern Sie den/die ursprünglichen Autor(en) und die Quelle ordnungsgemäß nennen, einen Link zur Creative Commons Lizenz beifügen und angeben, ob Änderungen vorgenommen wurden.

Die in diesem Artikel enthaltenen Bilder und sonstiges Drittmaterial unterliegen ebenfalls der genannten Creative Commons Lizenz, sofern sich aus der Abbildungslegende nichts anderes ergibt. Sofern das betreffende Material nicht unter der genannten Creative Commons Lizenz steht und die betreffende Handlung nicht nach gesetzlichen Vorschriften erlaubt ist, ist für die oben aufgeführten Weiterverwendungen des Materials die Einwilligung des jeweiligen Rechteinhabers einzuholen.

Weitere Details zur Lizenz entnehmen Sie bitte der Lizenzinformation auf http://creativecommons.org/ licenses/by/4.0/deed.de. 